\title{
Éditorial
}

\section{Surfaces et interfaces - Indentation, rayages et abrasion}

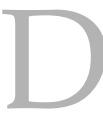

epuis sa création en 1997, la section ouest de la Société française de métallurgie et matériaux a organisé dans les différents pôles universitaires du grands ouest (Régions Bretagne, Pays de Loire et Poitou Charente) un colloque annuel sur des thématiques scientifiques touchant à la science des matériaux :

1997 : Nantes « Recristallisation »

1998 : Angers « Avancées des traitements et revêtements de surfaces »

1999 : Poitiers «Propriétés mécaniques des matériaux revêtus »

2000 : La Rochelle «Corrosion, dégradation et protection des matériaux »

2001 : St Nazaire «Contraintes résiduelles»

2002 : Rennes «Structures, microstructures et propriétés mécaniques des matériaux »

2003 : Lorient « Modélisation et simulation numérique en science des matériaux »

2004 : Nantes « Grandes vitesses et forts gradients »

2005 : Angers «Surfaces et interfaces » et journée thématique «Indentation, rayage et abrasion ».

Ces manifestations sont l'opportunité de rencontres privilégiées et d'échange entre chercheurs du secteur académique et ceux du milieu industriel travaillant dans le domaine de la «matériologie ».

Le colloque qui s'est tenu à l'ENSAM d'Angers le 16 et 17 mars 2005 avec le concours du programme PluriFormation de l'ENSAM, avait comme thème "Surfaces et Interfaces ». Au sein de ce cadre général, l'accent a plus particulièrement été mis sur les thématiques "Indentation, rayage et abrasion », s'inscrivant ainsi dans la continuité du séminaire " Nanoindentation » (ENSAM-Angers, 2003) et du mini-colloque " Micro-nanoindentation et plasticité » (Journées de la matière condensée, Nancy, 2004).

Rassemblés une nouvelle fois sur ces thèmes, et venant de communautés scientifiques différentes, près de 110 chercheurs se sont côtoyés lors de ces deux journées, leur conférant ainsi un fort succès.

À l'interface de la physique et de la mécanique, de l'instrumentation et de la simulation numérique, de la recherche académique et des développements industriels, les 18 exposés présentés, ont montré la diversité et la pluridisciplinarité des travaux développés actuellement dans ces thèmes.

Le présent numéro se veut le reflet de cette manifestation. La sélection d'articles que nous proposons donne un éclairage sur les sujets d'importance dans le domaine de la mécanique du contact, mettant en perspective avec un regard critique les résultats actuels, et présentant aussi des travaux récents tant expérimentaux que de simulation numérique.

L'article de M.M. Chaudhri expose tout d'abord les questions importantes et fondamentales que soulèvent les essais d'indentation. Il revient avec intérêt sur les concepts de taux de déformation, module réduit avant $\mathrm{d}$ 'insister sur la difficulté de l'étude des films minces. Ce thème est largement abordé tant expérimentalement que théoriquement dans l'ensemble du présent ouvrage. Un large éventail de matériaux (métaux, verres, semiconducteurs) est présenté par les auteurs rappelant la grande popularité de l'essai d'indentation tant dans l'industrie que dans le monde académique.

Nous souhaitons remercier l'ensemble des auteurs et rapporteurs pour la qualité de leur travail et vous souhaitons bonne lecture.

S. Benayoun, E. Le Bourhis 